\title{
Gestión De La Investigación En Las Universidades Publicas Del Perú: Avances Desde La Implementación De La Ley Universitaria 30220
}

DOI: $10.46932 / \mathrm{sfjdv} 1 \mathrm{n} 4-011$

Received in: August 1st, 2020

Accepted in: September 30th, 2020

\author{
Victoria Puente de la Vega Aparicio ${ }^{1}$ \\ Universidad Nacional de San Antonio Abad del Cusco-Perú. \\ E-mail: victoria.puentedelavega@unsaac.edu.pe
}

\begin{abstract}
Research is one of the great concerns of Latin American universities. In this context, the objective of this paper is to carry out an analysis of the actions implemented by the Vice-Rectorate for Research of one of the most representative Universities of Peru, such as the National University of San Antonio Abad del Cusco, within the framework of the application process of the New University Law No. 30220 promulgated in 2016, which emphasizes research as one of the substantial functions for university accreditation and quality. The document refers to the management of the research expressed in the regulatory changes, the modifications in the research policy, the financing, the research lines, the research teachers, the promotion of internships and mobilizations and the publication of articles and scientific journals. The progress achieved in the five years of the new university law is significant compared to previous decades.
\end{abstract}

Keywords: Research management, research policies, research lines, research teachers.

\section{INTRODUCCIÓN}

El presente trabajo analiza la gestión de la investigación en la Universidad Pública Peruana a través de la Universidad Nacional de San Antonio Abad del Cusco (UNSAAC), en el marco de la Nueva Ley Universitaria $\mathrm{N}^{\circ} 30220$ promulgada el 8 de julio del 2014. La UNSAAC es históricamente la 2da universidad más antigua del Perú y una de las 10 universidades más antiguas de Latinoamérica ${ }^{2}$, cumple actualmente 327 años de creación y se encuentra en franco proceso de modernización y adecuación a las nuevas tecnologías de información y comunicación.

La Nueva Ley Universitaria 30220 ha significado un cambio en el desarrollo de las universidades Públicas del Perú, al haber modificado aspectos sustanciales de la actividad universitaria, entre los cuales destacan: el énfasis dado a la calidad de las instituciones universitarias, el fortalecimiento de la

1 Docente Principal de la Facultad de Ciencias Económicas, Administrativas y Contables de la UNSAAC y Directora del Consejo de Unidades de Investigación de la UNSAAC.

2 El Sistema Universitario Peruano creció significativamente en los últimos 45 años. En 1969, existían en el Perú 22 universidades nacionales y 12 universidades privadas. En 1983, al promulgarse la ley 23733, existían 36 universidades. A inicio de 1990 solo existían 49 universidades entre públicas y privadas. En el 2015 existían 132 universidades, de las cuales 42 eran universidades públicas y 90 universidades privadas. De las 80 universidades creadas entre 1990 y el 2015 , el $83 \%$ son privadas. 
actividad investigativa, el reconocimiento de la responsabilidad social universitaria, la acreditación periódica, la inclusión de las universidades en el ámbito del Ministerio de Educación, entre otros. ${ }^{3}$

Este nuevo marco normativo universitario ha implicado especialmente una dinamización de la investigación, se han creado los Vicerrectorados de Investigación y consecuentemente existe una mayor presencia de investigadores en el diseño de las políticas, planes, programas y líneas de investigación, así como en la calificación de los proyectos de investigación con participación de pares o instituciones externas a la universidad; la asignación de fondos concursables para el financiamiento de proyectos de investigación apoyados principalmente por el canon gasífero y minero.

En menos de 5 años, la UNSAAC, ha normalizado su actividad científica con instrumentos normativos que guían y regulan las investigaciones, ha firmado varios convenios con entidades gubernamentales y privadas para garantizar la calidad de las investigaciones, como es el caso del Consejo Nacional de Ciencia, Tecnología e Innovación (CONCYTEC) y la Red De Investigación, Desarrollo e Innovación (RED IDi); ha promovido la creación de plataformas destinadas a la información y acceso a la investigación; está impulsado significativamente la movilidad y pasantías de docentes y estudiantes investigadores; está apoyando la realización de tesis de pre y post grado, así como la publicación de artículos científicos, entre otros.

Los resultados de esta nueva etapa en la vida universitaria pública son aún insuficientes, sin embargo, es innegable que se están poniendo las bases para el desarrollo de una actividad científica universitaria sostenible, responsable y sobre todo organizada y financiada para hacer frente a los complejos problemas sociales, económicos y ambientales de carácter regional, así como frente al desarrollo de las tecnologías de información y comunicación.

\section{DESARROLLO}

\subsection{CAMBIOS NORMATIVOS}

\subsubsection{Ley 30220}

La nueva ley universitaria promulgada en julio del 2014, constituye un cambio importante en el devenir universitario peruano, particularmente en el campo de la investigación, debido a que el artículo $63^{\circ}$ de la ley abre la posibilidad de contar con Vicerrectorados de Investigación, señalando en el art. 65.2, las atribuciones del Vicerrectorado de Investigación. Destina un capítulo (capítulo VI) a la

Las dos penúltimas leyes universitarias en el Perú, la Ley 17437 promulgada en febrero de 1969 y la Ley 23733 promulgada el 9 de diciembre de 1983, no promovieron el desarrollo de la investigación, pese a considerarla una función fundamental de la actividad universitaria, no hubo una verdadera política de promoción de la investigación que se reflejara en el Presupuesto General de la República. La Ley 23733 fue una de las normas universitaria que más tiempo permaneció en la legislación peruana, desde 1983 hasta el 2016. 
investigación, en el que no solamente la reconoce como función esencial y obligatoria de la universidad para responder a través de la producción de conocimientos y desarrollo de tecnologías a las necesidades de la realidad nacional, sino que además abre la posibilidad de crear fondos de investigación y sobre todo de otorgar bonificaciones a los docentes investigadores, que posteriormente se materializó en una norma específica*. En resumen, esta ley, otorga muy claras atribuciones a los Vicerrectorados de Investigación, reconociendo su rol central y su alta responsabilidad en materia de investigación, encargándole además la orientación, coordinación y organización de proyectos y actividades de investigación que se desarrollan a través de las unidades académicas; la organización y difusión del conocimiento; la promoción y aplicación de los resultados de las investigaciones y la transferencia tecnológica a la sociedad, mediante la integración de los tres actores más importantes para el desarrollo social: la universidad, la empresa y el Estado. Finalmente, la Ley Universitaria 30220 integra dentro del ámbito de la investigación de las universidades: la coordinación con entidades públicas y privadas para la atención de investigaciones, las incubadoras de empresas, el reconocimiento de los derechos de autor y patentes y la promoción de centros de producción de bienes y servicios, cuyas utilidades constituirán recursos destinados prioritariamente a la investigación.

\subsubsection{Creación del Vice Rectorado de Investigación en la UNSAAC (2011)}

El 28 de octubre del 2011, mediante Resolución de Asamblea Universitaria $\mathrm{N}^{\circ}$ 010-2011 UNSAAC, La Universidad Nacional de San Antonio Abad del Cusco, creo el Vicerrectorado de Investigación, tres años antes de la promulgación de la Ley 30220, modificando automáticamente su propio Estatuto. Durante los años 2011 al 2014, antes que se promulgara la Ley 30220, el Vicerrectorado de Investigación estuvo dedicado a modernizar y fortalecer su estructura y sus instrumentos normativos.

\subsubsection{Agenda Regional de Investigación (2015)}

En año 2015, la UNSAAC en coordinación con otras organizaciones de la Región Cusco, participaron en la elaboración de la Agenda Regional de Investigación, documento que fue aprobado el 23 de setiembre del año 2015, mediante ordenanza Regional N 100-2015-CR/GRC-CUSCO. Dicho documento constituye uno de los primeros esfuerzos regionales para promover la investigación así como uno de los primeros nexos entre la empresa, el Estado y la Universidad para responder a la problemática y las necesidades de investigación de la región del Cusco, habida cuenta que hasta esa fecha no existía una política ni estrategia que abordara la problemática de la investigación en el ámbito regional, tampoco una voluntad de cooperación interinstitucional ni recursos suficientes para promover la 
investigación en el ámbito regional. Dicha Agenda tiene como objetivo fundamental: orientar el desarrollo de la investigación para "generar conocimiento que contribuya a la solución de los problemas de la Región Cusco y al despliegue de sus potencialidades hacia un desarrollo humano y sustentable" (ARI, 2015, pág. 77).

Este importante documento establece las siguientes dimensiones y ejes temáticos de investigación: la dimensión social integrado por los ejes de: salud, educación, pobreza y equidad y cultura; la dimensión económica integrada por los ejes: sector agropecuario, turismo, energía y valor agregado; la dimensión ambiental integrado por el sector ambiental propiamente dicho y finalmente por las investigaciones transversales que son numerosas pero agrupadas en las áreas de: demografía, articulación regional, tecnologías de información y comunicación, gobernanza, escenarios climáticos e hidrológicos y gestión del riesgo de desastres. (ARI, 2015, págs. 126-130)

\subsubsection{Creación del CORCYTEC}

El 2 de Julio del año 2012, mediante Ordenanza № 30-2012-CR/GRC-CUSCO, El Gobierno Regional del Cusco, crea el CORCYTEC como organismo descentralizado del gobierno regional cuyo objetivo fundamental es contar con "un espacio de concertación entre las instituciones públicas, privadas y sociedad civil que impulsen y canalicen el desarrollo de la ciencia, tecnología e innovación y enriquezca las capacidades para generar y utilizar los conocimientos científicos y tecnológicos en el desarrollo de nuestra región" (Gobierno Regional Cusco, 2012).

Este organismo, estuvo conformado por los representantes de las instituciones de la región ,no obstante significar una excelente iniciativa, el CORCYTEC, no tuvo actividad ni pudo desarrollarse como se esperaba entre los años 2012 al 2019; probablemente debido a la falta de apoyo financiero; poca o nula participación de las instituciones que la conformaron, carencia de una estructura orgánica que le de soporte, ausencia de empresas privadas y desarticulación respecto a los programas y actividades del Consejo Nacional de Ciencia y Tecnología (CONCYTEC), que parece no tomar en cuenta el desarrollo de estas iniciativas regionales.

\subsection{MODIFICACIONES EN LA POLÍTICA DE INVESTIGACIÓN}

\subsubsection{Cambios estructurales y organizacionales}

El Vicerrectorado de Investigación de la UNSAAC, en estricto cumplimiento a la Ley Universitaria 30220 elabora su Estatuto en el que establece una amplia y extensa normatividad (artículos $132^{\circ}$ al $155^{\circ}$ ), además de otorgarle cinco órganos y 3 direcciones de investigación, con lo cual se impulsó de manera significativa la investigación en la UNSAAC. 
Entre los órganos de investigación se encuentran: el Vicerrectorado de Investigación; el Consejo de Unidades de Investigación; los Institutos de Investigación Interdisciplinaria; los Centros de Investigación y los Centros de Investigación de estudiantes. Entre las direcciones de Investigación se encuentran: La Dirección de Gestión de la Investigación; la Dirección de Emprendimiento y Gestión y la Dirección de Innovación y Transferencia.

\section{a. Vicerrectorado de Investigación}

El Vicerrectorado de Investigación es el organismo de más alto nivel en el ámbito de la investigación de la UNSAAC, está encargado de "dirigir y ejecutar la política general de investigación en la universidad y supervisar las actividades de investigación con la finalidad de garantizar la calidad de las mismas y su concordancia con la misión y metas establecidas por el Estatuto de la Universidad" (Art. 65.2.1. y 65.2.2. de la Ley $\mathrm{N}^{\circ}$ 30220). Sus funciones y atribuciones están determinadas en el artículo 65.2 de la Ley 30220.

\section{b. Consejo de Unidades de Investigación}

El Consejo de Unidades de Investigación es el órgano del Vicerrectorado de Investigación conformado por los Directores de cada Unidad de Investigación y los Directores de los Institutos de Investigación Interdisciplinaria. Se reúne al menos una vez al mes o por convocatoria del Director del Consejo de las Unidades de Investigación. Sus funciones están establecidas en el artículo 136.2 del Estatuto de la UNSAAC.

\section{c. Unidades de Investigación}

Las unidades de investigación son órganos de investigación encargados de integrar las actividades de investigación de cada Facultad. Está conformada por los centros de investigación de las Escuelas Profesionales. Cada Unidad de Investigación tiene un Comité Directivo conformado por los Directores de los Centros de Investigación de la Facultad. Sus funciones y atribuciones están establecidas en el art. 140.4 del Estatuto de la UNSAAC.

\section{d. Centros de Investigación}

Los centros de Investigación son las “células básicas donde se forman y desenvuelven las comunidades científicas dentro de la UNSAAC y donde se diseñan, evalúan y ejecutan los proyectos de investigación”. Posee autonomía académica, organizacional y de gestión de sus recursos económicos. Sus Funciones y atribuciones está establecidas en el artículo $141^{\circ}$ del Estatuto de la UNSAAC. 


\section{e. Centros de investigación de estudiantes}

Los Centros de Investigación de estudiantes "tienen la finalidad de promover la iniciación científica en todas las modalidades y de generar nuevos conocimientos que solucionen los problemas regionales y nacionales". Están "conformados por estudiantes, asesores docentes, asesores externos, cesantes y jubilados". Sus alcances están determinados en el artículo $142^{\circ}$ del Estatuto de la UNSAAC.

\section{f. Institutos de Investigación Interdisciplinaria}

Los Institutos de Investigación Interdisciplinaria son entidades "donde se forman y desenvuelven las comunidades científicas conformadas por investigadores de las diferentes facultades de la UNSAAC y donde se diseñan, evalúan y ejecutan los proyectos de investigación interdisciplinarios. Dependen del Vicerrectorado de Investigación. Sus características específicas y alcances están determinados en el artículo $143^{\circ}$ del Estatuto de la UNSAAC.

\section{g. Dirección de Gestión de la Investigación}

La Dirección de Gestión de la Investigación es un órgano de línea del Vicerrectorado de Investigación "encargado de fortalecer las capacidades de investigación de la comunidad universitaria y del cumplimiento eficaz y eficiente del ciclo de la investigación”. Sus funciones están establecidas en el artículo $137^{\circ}$ del Estatuto de la UNSAAC.

\section{h. Dirección de Innovación y transferencia}

La Dirección de innovación y transferencia, es un órgano de línea del Vicerrectorado de Investigación "encargado de visibilizar a nivel nacional e internacional los resultados de las investigaciones de la UNSAAC". Las funciones de este órgano están establecidas en el artículo 138 del Estatuto de la UNSAAC.

\section{i. Dirección de Emprendimiento y Gestión}

La Dirección de Emprendimiento y Gestión es un órgano “encargado de la formación de cultura empresarial entre los estudiantes y la comunidad universitaria”. Sus funciones están establecidas en el artículo $139^{\circ}$ del Estatuto de la UNSAAC.

Esta estructura orgánica le otorga un mayor peso y presencia a la investigación en el conjunto de la actividad universitaria, además de asignarle atribuciones para incorporar la participación del Estado, la empresa y la Universidad en la investigación, así como la coordinación con entidades públicas y 
privadas, la supervisión de las incubadoras de empresas, los derechos de autor y las patentes y los centros de producción de bienes y prestación de servicios.

\subsubsection{Aprobación de instrumentos normativos:}

Entre los años 2011 y 2019, y particularmente a partir de la nueva Ley Universitaria $\mathrm{N}^{\circ} 30220$, el Vicerrectorado de Investigación de la UNSAAC formuló un conjunto de instrumentos normativos para impulsar la investigación universitaria. Entre estos instrumentos destacan los siguientes:

1. Reglamento para la creación, adecuación y actividades de los Centros de Investigación, aprobado mediante Resolución N CU-0240-2017-UNSAAC el 8 de Junio del 2017.

2. Reglamento del Docente Investigador, aprobado mediante Resolución N ${ }^{\circ}$ CU-0202-2017UNSAAC el 10 de mayo del 2017.

3. Plan General de Fortalecimiento de la Investigación en la UNSAAC 2016-2020, aprobado mediante Resolución N CU-561-2018-UNSAAC el 23 de noviembre del 2018.

4. Reglamento del Repositorio Institucional Digital de la UNSAAC, aprobado mediante Resolución $\mathrm{N}^{\circ}$ CU-0201-2018-UNSAAC de fecha 26 de abril del 2018.

5. Reglamento para el uso del sistema anti-plagio, aprobado mediante Resolución $\mathrm{N}^{\circ}$ CU-03822018-UNSAAC de fecha 6 de agosto del 2018.

6. Líneas de Investigación UNSAAC - 2021, aprobado mediante Resolución № CU-097-2018UNSAAC de fecha 8 de marzo del 2018.

No son los únicos instrumentos normativos formulados pero son los más importantes y los que están permitiendo la gestión de la investigación en la UNSAAC. Gran parte de estos instrumentos normativos son totalmente nuevos, es decir, no existían en la gestión anterior ni en los alcances de la Ley Universitaria 27733. Sin embargo, esta nueva reglamentación y marco normativo en materia de investigación no solo se debe a los efectos de la Ley 30220, sino también a la madurez alcanzada por los gestores de investigación y docentes investigadores que desean contar con medios, instrumentos, métodos, canales y apoyos a sus proyectos de investigación y a su actividad investigativa en general, buscan formas de apoyo y coordinación de esfuerzos universitarios para el desarrollo de la investigación en la UNSAAC.

\subsubsection{Creación de plataformas de acceso a la investigación}

Hasta antes de la promulgación de la Ley 30220, la UNSAAC carecía de una plataforma virtual de acceso a la información, difusión, comunicación y participación en materia de investigación. Hoy, la 
UNSAAC cuenta un portal exclusivamente dedicado a la investigación en sus distintas áreas y actividades, al que se ha denominado en idioma quechua como: "Yachayninchis Wiñarinanpaq" cuya traducción significa "Para que nuestro conocimiento crezca". Este portal contiene información acerca de las funciones y organización del Vicerrectorado de Investigación, unidades y centros de investigación, incubadora de empresas, Innovación y transferencia de conocimientos, convocatorias a concursos, noticias relacionadas a la investigación, difusión de eventos de investigación, publicación de instrumentos normativos, así como documentación de interés científico e investigativo, a los que puede acceder cualquier persona de la comunidad universitaria y de la sociedad.

No obstante, aún falta desarrollar este ámbito. Una de las áreas que se encuentra en su fase incipiente es el repositorio de investigaciones, tesis de pre y post grado, artículos científicos, revistas científicas, etc. Actualmente se viene trabajando en ese sentido y poco a poco se ha iniciado con la difusión de tesis de pre y post grado, primeras revistas y artículos científicos. Sin embargo falta elevar el nivel de calidad de las publicaciones, promover el reconocimiento e indización de las revistas, mejorar el control de calidad en los procesos de investigación, el control en la originalidad de los trabajos de investigación, el control de calidad en la redacción de los trabajos de investigación, etc. Otro aspecto que requiere desarrollar es la información referida a los proyectos de investigación, el estado de avance, los resultados alcanzados y el cierre de los mismos. Por otro lado, aún se carece de un canal de acceso a las redes mundiales de ciencia e investigación en los distintos idiomas, los cuales necesitan de acuerdos o convenios internacionales, o en su defecto de adquisiciones comerciales de derechos de acceso. Estas plataformas permitirían a profesores, estudiantes, investigadores y tesistas, acceder en línea a revistas científicas y resultados de investigaciones arbitradas y actualizadas en todas las áreas del conocimiento científico.

\subsubsection{Convenios y alianzas para impulsar la investigación}

Se debe resaltar que en el proceso de adecuación a la Ley Universitaria 30220 con fines del licenciamiento institucional y la acreditación se destaca dos de las más importantes alianzas interinstitucionales en materia de gestión de la investigación:

\section{a) Convenio UNSAAC-CONCYTEC}

El 28 de noviembre del 2016, la UNSAAC firmó un convenio con el CONCYTEC con el objeto de establecer el marco general de cooperación interinstitucional, pautas y mecanismos que permitan el desarrollo de actividades de cooperación, promoción y financiamiento en materia de ciencia, tecnología 
e innovación, para el fortalecimiento de las capacidades para la investigación científica, desarrollo tecnológico e innovación tecnológica en la Universidad. La duración de dicho convenio es de 3 años.

\section{b) Convenio UNSAAC-Red IDI}

En el segundo semestre del 2018, la UNSAAC firma un convenio de interacción científica con la Red de Investigación, Desarrollo e Innovación (RED-IDi) que congrega a las más importantes universidades públicas y privadas del Perú ${ }^{4}$ y con la cual ha comenzado a operar en la calificación de los proyectos de investigación a través de los fondos concursables canon. La RED IDi es una asociación sin fines de lucro creada el año 2007, formalizada como asociación sin fines de lucro el 2009. Tiene como visión "ayudar a consolidar el desarrollo económico y social del país, fortaleciendo el nexo entre la Universidad y la empresa, fomentando el uso de herramientas de investigación científica y su aplicación para satisfacer necesidades de la sociedad, con los más altos estándares éticos y profesionales”. (RED IDi, 2019)

\subsection{FINANCIAMIENTO DE LA INVESTIGACIÓN}

\subsubsection{Fuentes de financiamiento}

La UNSAAC posee dos fuentes de financiamiento destinadas a la investigación: El Fondo Especial de Desarrollo Universitario (FEDU) creado por Ley $\mathrm{N}^{\circ} 25203$ y los fondos Canon creado mediante Ley $\mathrm{N}^{\circ} 27506$.

\section{a) El Fondo Especial de Desarrollo Universitario (FEDU)}

El FEDU fue creado a través de la Ley N² 25203 en febrero de 1990, su objetivo es contribuir al financiamiento de la investigación en Universidades Públicas del país y cada universidad por reglamento regula la presentación, registro, ejecución, monitoreo y evaluación de los Proyectos de Investigación. Estos fondos son distribuidos entre las universidades públicas del país de acuerdo a los criterios establecidos por la SUNEDU (antes Asamblea Nacional de Rectores). En el caso de la UNSAAC los fondos FEDU son asignados como incentivos a la investigación por un monto de 300 soles mensuales a todos los docentes ordinarios que conforman los equipos y elaboran proyectos bianuales. El reconocimiento institucional (Resolución del VRIN) beneficia a los docentes en los procesos de ascensos y ratificación con puntajes importantes. Es necesario indicar que la ausencia de

\footnotetext{
${ }^{4}$ Integran esta Red la Universidad Nacional Mayor de San Marcos, la Universidad Nacional de Ingeniería, la Universidad Nacional Agraria La Molina, la Pontificia Universidad Católica del Perú, la Universidad Peruana Cayetano Heredia, la Universidad Nacional San Agustín de Arequipa y la Universidad Nacional de San Antonio Abad del Cusco.
} 
rigor científico de estos proyectos de investigación no ha sido ni es favorable a la producción intelectual de la institución.

\section{b) Fondo Canon}

Este fondo está regulado por la Ley $\mathrm{N}^{\circ}$ 27506, aprobado en el mes de Julio del 2001. Durante los últimos años fue motivo de varias modificaciones, especialmente en la distribución de dichos fondos. Del $100 \%$ de estos fondos el 25\% corresponde al Gobierno Regional del Cusco, el que asigna el $20 \%$ a las universidades. Se debe señalar que a partir del 2014 parte de estos fondos son compartidos con la Universidad Nacional Intercultural de Quillabamba, que son las entidades universitarias públicas del departamento del Cusco. La Ley señala que estos fondos deben ser "exclusivamente destinados a la inversión en investigación científica y tecnológica que potencien el desarrollo Regional”, además de incorporar tales transferencias financieras en sus respectivos presupuestos como "donaciones y transferencias". (Art. 6.2 de la Ley N²7506).

\subsubsection{Recursos para la investigación científica y tecnológica}

Durante los años 2017-2019, los recursos determinados por concepto de Canon y sobre Canon que debieron ser transferidos a la UNSAAC alcanza a un total de 107 millones 248 mil, 374 soles. Estos montos experimentan un crecimiento anual promedio del 18.5\%. Se debe tomar en cuenta que los recursos provenientes del canon gasífero es el más significativo, llegando a representar más del 90\% de los recursos totales provenientes del canon.

Tabla 1: Recursos Determinados por Canon y sobre Canon 2017-2019

\begin{tabular}{|c|c|c|c|}
\hline Fuente & Canon & Monto S/. & Total S/. \\
\hline \multirow{4}{*}{2017} & Minero & $978,839.00$ & \multirow{4}{*}{$29^{\prime} 867,200$} \\
\hline & Gasífero & $28^{\prime} 674,834.00$ & \\
\hline & Hidroenergético & $212,316.00$ & \\
\hline & Forestal & $1,211.00$ & \\
\hline \multirow[t]{4}{*}{2018} & Minero & $886,972.00$ & \multirow{4}{*}{$35^{\prime} 401,846$} \\
\hline & Gasífero & $34 ' 193,944.00$ & \\
\hline & Hidroenergético & $319,719.00$ & \\
\hline & Forestal & $1,211.00$ & \\
\hline \multirow[t]{4}{*}{2019} & Minero & 3'528,189.00 & \multirow{4}{*}{$41^{\prime} 979,328$} \\
\hline & Gasífero & $37^{\prime} 987,117.00$ & \\
\hline & Hidroenergético & $462,811.00$ & \\
\hline & Forestal & $1,211.00$ & \\
\hline \multicolumn{3}{|c|}{ Total } & $107^{\prime} 248,374$ \\
\hline
\end{tabular}

Fuente: Vicerrectorado de Investigación UNSAAC 


\subsubsection{Proyectos de investigación financiados con los fondos canon}

De acuerdo a los datos del Vicerrectorado de Investigación de la UNSAAC, entre los años 2011 y 2017 se financiaron 86 proyectos de investigación con un monto total de 84 millones 280 mil 428.81 soles, siendo los años 2013 y 2017 los de mayor número de proyectos de investigación aprobados. A partir del año 2017, los fondos son concursables con la intervención del CONCYTEC, teniendo como monto máximo de financiación la suma de 500 mil soles por proyecto de investigación. El 2018 no hubo convocatorias y el 2019 las convocatorias para proyectos de investigación están en pleno proceso de calificación.

Tabla 2: Número total de proyectos de investigación y montos aprobados 2011-2017

\begin{tabular}{|c|c|c|}
\hline Año & $\begin{array}{c}\mathbf{N}^{\circ} \text { de Proyectos de } \\
\text { investigación aprobados }\end{array}$ & Monto aprobado en soles \\
\hline 2011 & 12 & $17^{\prime} 024,086.52$ \\
\hline 2012 & 2 & $2 ' 557,524.00$ \\
\hline 2013 & 26 & $31^{\prime} 761,910.48$ \\
\hline 2014 & 6 & $10^{\prime} 269,793.05$ \\
\hline 2015 & 7 & $9 ' 345,217.95$ \\
\hline 2016 & 1 & $188,821.00$ \\
\hline 2017 & 32 & 13 '133,075.81 \\
\hline Total & $\mathbf{8 6}$ & $\mathbf{8 4 , 2 8 0 , 4 2 8 . 8 1}$ \\
\hline
\end{tabular}

\subsection{LÍNEAS DE INVESTIGACIÓN}

En el mes de noviembre del 2017, luego de un poco más de tres años de aprobada la nueva Ley Universitaria 3220, la UNSAAC, formula un documento denominado Líneas de Investigación UNSAAC 2018-2021, el mismo que intenta responder no solamente a los requerimientos de la Ley sino a las necesidades de la comunidad científica universitaria, que demandaba desde hace muchos años, pautas para orientar sus investigaciones dentro del marco de las necesidades sociales, las políticas de investigación universitarias y los nuevos avances de los conocimientos e instrumentos tecnológicos.

Bajo la coordinación del Vicerrectorado de Investigación a través de su Dirección de Gestión de la Investigación se realizaron talleres de trabajo, reuniones de coordinación y discusión de varias propuestas. Finalmente se identificaron siete áreas del conocimiento y 67 líneas de investigación, para evidencia se presentan las líneas de investigación del área del Ciencias Sociales, Económicas y Humanidades $(\mathrm{CEH})$ : 
Tabla 3: Líneas de Investigación UNSAAC 2018-2021

\begin{tabular}{|c|c|c|}
\hline $\begin{array}{c}\text { Áreas del } \\
\text { conocimiento }\end{array}$ & Código & Línea de Investigación \\
\hline \multirow{24}{*}{$\begin{array}{l}\text { Ciencias sociales, } \\
\text { económicas y } \\
\text { humanidades - } \mathrm{CEH}\end{array}$} & $\mathrm{CEH}-01$ & Administración y gestión pública y privada \\
\hline & $\mathrm{CEH}-02$ & Análisis de la filosofía regional \\
\hline & $\mathrm{CEH}-03$ & Arqueología histórica \\
\hline & CEH-04 & Auditoría, tributación e información financiera \\
\hline & CEH-05 & Comunicación educativa \\
\hline & CEH-06 & Comunicación social y periodismo \\
\hline & CEH-07 & Desarrollo económico local \\
\hline & CEH-08 & Economía social y educativa \\
\hline & CEH-09 & Emprendimiento, marketing y PYMES \\
\hline & CEH-10 & Estado, instituciones públicas y comunicación política \\
\hline & CEH-11 & Estudios históricos \\
\hline & CEH-12 & Estudios interculturales \\
\hline & CEH-13 & Ética, derechos humanos y cultura de paz \\
\hline & CEH-14 & Familia y educación \\
\hline & CEH-15 & Formación académica y currículum \\
\hline & CEH-16 & Gestión del desarrollo turístico \\
\hline & CEH-17 & Gestión educativa \\
\hline & CEH-18 & Impactos socioculturales y ambientales del turismo \\
\hline & CEH-19 & Propuesta formativa \\
\hline & CEH-20 & Psicología clínica y educativa \\
\hline & CEH-21 & Psicología organizacional y laboral \\
\hline & CEH-22 & Responsabilidad social empresarial \\
\hline & CEH-23 & Sistema bancario y financiero \\
\hline & CEH-24 & Sistemas de comunicación andino-amazónicos \\
\hline
\end{tabular}

Fuente: Documento: Líneas de Investigación - UNSAAC

Si bien este documento ha sido resultado de un trabajo de coordinación académica y revisión bibliográfica, con participación de autoridades y docentes de diversas especialidades y constituye un gran avance en la normalización y sistematización de las investigaciones en la UNSAAC, aún adolece de una excesiva generalidad que no resulta operativa para guiar y conducir las investigaciones en la universidad. Más que líneas de investigación en muchos casos se señalan disciplinas o sub-disciplinas del conocimiento, ejemplo, casi todas las líneas del área Ciencias Sociales, Económicas y Humanidades son disciplinas o subdisciplinas, las que no reflejan suficientemente la problemática social y económica regional ni el sentido multidisciplinario, interdisciplinario y transdisciplinario que se debería enfatizar en la universidad dada la complejidad de los problemas sociales.

\subsection{DOCENTES INVESTIGADORES}

La Ley 30220 en su artículo $86^{\circ}$ señala la existencia de los docentes investigadores y define como docente investigador a "aquel que se dedica a la generación de conocimiento e innovación, a través de la investigación. Es designado en razón de su excelencia académica. Su carga lectiva será de un (1) curso por año y tiene una bonificación del $(50 \%)$ de sus haberes totales. Está sujeto al régimen especial que la universidad determine en cada caso. El Vicerrectorado de Investigación o la autoridad competente 
evalúa cada dos años la producción de los docentes, para su permanencia como investigador en el marco de los estándares del Sistema Nacional de Ciencia, Tecnología e Innovación Tecnológica (SINACYT)”.

Adicionalmente el CONCYTEC, órgano rector del SINACYT estableció a través de la Resolución de Presidencia $\mathrm{N}^{\circ}$ 198-2017-CONCYTEC-P un conjunto de estándares para la calificación y registro de investigadores, el mismo que está constantemente sujeto a evaluación por parte del CONCYTEC, de acuerdo a la verificación de los propios datos proporcionados tanto por los investigadores y las universidades. El inciso 7.2 del artículo $7^{\circ}$ de la Resolución de Presidencia N 198-2017 señala además que "las Universidades solo pueden considerar docente investigador a aquella persona natural que cuente con la calificación vigente de Investigador otorgada por CONCYTEC y cumpla con los estándares del SINACYT definidos por el CONCYTEC.

En este marco, los docentes dedicados a la investigación científica de la Universidad Nacional de San Abad del Cusco iniciaron un proceso de impulso, calificación y reconocimiento de sus actividades científicas de acuerdo a los estándares señalados por el CONCYTEC. El incremento gradual de los docentes REGINA: 10(2017) ,18 (2018), 27(2019) es aún bajo en relación a las estadísticas del total de 2534 investigadores REGINA registrados en el CONCYTEC.

El Vicerrectorado de Investigación de la UNSAAC debe seguir impulsando la producción científica y publicaciones de sus docentes, tanto nombrados como contratados, hasta alcanzar cuando menos a un $20 \%$ de sus docentes nombrados. Para ello, se necesita seguir desarrollando la política de promoción de la investigación, ampliando y mejorando las plataformas de información y gestión de la investigación en línea y propiciando el acceso a la información y resultados de investigaciones a nivel mundial en todos los idiomas.

\subsection{PUBLICACIÓN DE ARTÍCULOS DE INVESTIGACIÓN}

Al $1^{\circ}$ de Marzo del 2018 y de acuerdo a los datos publicados por el Vicerrectorado de la UNSAAC en su documento denominado Líneas de Investigación (UNSAAC, 2017), el número de artículos publicados en revistas indizadas por los 18 docentes investigadores de la UNSAAC con los cuales contaba hasta ese momento alcanzaba solamente a un total de 63 artículos científicos. Entre las plataformas de publicación utilizadas por estos investigadores se encuentran: SCOPUS, sciELO, Web of Science y Medline.

La baja tasa de publicaciones de artículos científicos en la UNSAAC en relación a sus pares nacionales e internacionales es insuficiente dada la importancia de la universidad, la cantidad de docentes nombrados, el número de facultades y escuelas profesionales existentes Consciente de esta realidad, el Vicerrectorado de Investigación de la Universidad viene actualmente impulsando un 
conjunto de estrategias para dar soporte a la investigaciones, a la redacción de artículos científicos, a la potenciación o creación de revistas científicas virtuales y a la visibilización de la producción científica. Algunas de estas estrategias son: la realización de cursos de capacitación docente en redacción científica, la asignación de fondos concursables para la publicación de artículos científicos con afiliación de la UNSAAC, hasta por 8,000 soles por artículo.

\subsection{REVISTAS CIENTÍFICAS}

La carencia de revistas científicas universitarias en cada una de las facultades es otra de las realidades más atingentes y clamorosas que experimenta la universidad. Actualmente se cuenta solamente con 4 revistas no indizadas de carácter general, es decir, no están dedicadas exclusivamente a la difusión científica, sus ediciones están pensadas para su publicación en papel y a corto tiraje. Entre las revistas que han mantenido cierta regularidad se encuentran:

\section{- Revista El Antoniano}

Revista académica de carácter general, publicada en papel, actualmente se encuentra en el $\mathrm{N}^{\circ}$ 131 y su última publicación la realizó en agosto del 2017, se encuentra discontinuada.

- Revista de la Facultad de derecho y Ciencias Políticas

Revista promovida por la Facultad de Derecho y Ciencias Políticas, se encuentra en el número 10 y su última publicación fue realizada el año 2018.

\section{- Revista Cambio Climático en los Andes Tropicales}

Revista patrocinada por el Vicerrectorado de Investigación de la Universidad Nacional de San Antonio Abad del cusco, edición impresa en papel con carácter semestral. Publicó hasta el Volumen 2 Número 1, su última publicación fue en el año 2015. Publica artículos de investigación que tratan sobre los cambios del régimen de precipitación y temperatura ambiental que incrementa la fragilidad y susceptibilidad del ecosistema a nivel nacional e internacional.

\section{- La Revista "Cantúa”}

Revista de Ciencias Biológicas. Versión Impresa en papel con carácter semestral, se encuentra en el $\mathrm{N}^{\circ} 14$, su última publicación fue realizada en el 2015, se encuentra descontinuada. 
Se considera importante impulsar la creación de Revistas Científicas en cada Facultad, para cuyo efecto se necesitarían cuatro aspectos fundamentales. a) La creación de comités editoriales en cada Facultad, b) Intensificación de capacitación a los docentes en el diseño de Revistas Científicas y redacción de artículos científicos. c) Creación de plataformas virtuales para la publicación de las revistas y artículos científicos en línea. d) La calificación de los artículos científicos por pares externos.

\subsection{REPOSITORIO DE TESIS}

La publicación en línea de las tesis de pre y post grado fue una práctica instaurada desde hace varias décadas por las universidades más avanzadas de Estados Unidos y Europa.. A partir de las década del 90 y coincidente con el desarrollo y utilización masiva del internet y uso de medios digitales para el archivo de datos, la práctica de publicación de investigaciones y tesis de pre y post grado a través de repositorios electrónicos se hicieron más populares en casi todas las universidades del mundo. La UNSAAC, empezó a utilizar su portal de internet para estas publicaciones a partir del año 2010, pero fue recién a partir del año 2015 que intensificó tales publicaciones. Actualmente (a julio del 2019) ha logrado publicar en la Red un total de 3037 investigaciones, de los cuales 501 son tesis de Post grado y 2494 tesis de pre grado, además de 32 artículos de revistas externas con filiación UNSAAC.

Tabla 4: Publicaciones en el Repositorio UNSAAC Período 2010-2019

\begin{tabular}{|c|c|c|}
\hline & Tipo de tesis y Facultad & $\mathbf{N}^{\circ}$ \\
\hline \multicolumn{3}{|c|}{ Tesis de Post Grado } \\
\hline 7. & Doctorados & 20 \\
\hline 8. & Maestrías & 481 \\
\hline \multicolumn{3}{|c|}{ Tesis de Pre Grado } \\
\hline 9. & Facultad de Arquitectura e Ingeniería Civil & 187 \\
\hline 10. & Facultad de Ciencias & 219 \\
\hline 11. & Facultad de Ciencias Administrativas, Contables, Económicas y Turismo & 435 \\
\hline 12. & Facultad de Ciencias Agrarias & 344 \\
\hline 13. & Facultad de Ciencias de la Salud & 501 \\
\hline 14. & Facultad de Derecho y Ciencias Sociales & 148 \\
\hline 15. & Facultad de Educación y Ciencias de la Comunicación & 222 \\
\hline 16. & Facultad de Ingeniería de Procesos & 49 \\
\hline 17. & Facultad de Ingeniería Eléctrica, Electrónica, Informática y Mecánica & 226 \\
\hline 18. & Facultad de Ingeniería Geológica, Minas y Metalurgia & 173 \\
\hline Artí & de Revistas Externas con Filiación UNSAAC & 32 \\
\hline & Total & 3,037 \\
\hline
\end{tabular}

Fuente: http://repositorio.unsaac.edu.pe/

Estas publicaciones presentan problemas en relación a criterios de: a) originalidad, b) calidad de presentación y argumentativa en los escritos y c) calidad en el manejo de los métodos, diseños y técnicas de investigación. Ello implica a su vez la necesidad de mejorar la calidad de las cátedras de formación, la formación científica y metodológica de los estudiantes y profesores. 


\subsection{PASANTÍAS Y MOVILIZACIONES CIENTÍFICAS}

La promoción de las pasantías y movilizaciones para la participación en eventos de investigación en calidad de ponentes o asistentes fue una de las preocupaciones del Vicerrectorado de Investigación desde el año 2017 en adelante. En el semestre 2017-I se autorización y financiaron 14 pasantías y movilizaciones por un monto de 204, 150.05 soles; el 2017-II se autorizaron y financiaron 17 pasantías y movilizaciones por un monto de 402,607.51 soles. "Las pasantías tuvieron un periodo de permanencia de una semana hasta tres meses y fueron realizadas en universidades de Argentina, México, Chile, Estados Unidos, Canadá, España, Gran Bretaña, Francia y Perú” (UNSAAC, 2019).

Las pasantías y movilizaciones dependerán de las vinculaciones académicas nacionales e internacionales que logren realizar los investigadores en el marco de las líneas de investigación, la permanente revisión y la vigilancia tecnológica en las diferentes áreas del conocimiento es un imperativo para cualquier organización y más aún para las universidades.

En la medida que la cooperación académica se amplié, las posibilidades de la movilizaciones y pasantías tanto para docentes y estudiantes será progresiva y se considera pertinente aplicar un mejor seguimiento de los resultados a fin de garantizar la efectividad en la difusión de los conocimientos adquiridos así como en la publicación de los artículos cuando se trata de pasantías mayores a 30 días.

\section{CONCLUSIONES}

3.1. Es innegable que La Ley Universitaria $N^{\circ} 30220$ aprobada el 2016 ha significado un viraje en la vida universitaria peruana, particularmente en el campo de la investigación, haciendo que la actividades de gestión de la investigación cobren una mayor relevancia para atender un crecimiento de orden cualitativo y cuantitativo en las investigaciones como consecuencia de las nuevas políticas de investigación.

3.2. Los cambios normativos expresados en la aprobación de la nueva Ley universitaria y la creación de los Vicerrectorados de Investigación en las universidades Públicas, aunado a los acuerdos regionales sobre investigación expresados en la creación del CORCYTEC y la formulación de la Agenda Regional de Investigación dieron un soporte jurídico y social al desarrollo de la investigación en la Universidad Nacional de San Antonio Abad del Cusco.

3.3. La reformulación del Estatuto de la UNSAAC a partir de la nueva Ley Universitaria, particularmente de los aspectos relacionados a la investigación, así como las modificaciones en la política de investigación de la UNSAAC ha encontrado respuesta en la comunidad universitaria, particularmente entre los docentes investigadores, quienes han acudido a las diferentes convocatorias impulsadas por el Vicerrectorado de Investigación. Si bien los 
resultados son aún insuficientes, se espera que el ritmo de crecimiento y desarrollo de la investigación en los próximos años sea mayor.

3.4. A partir del año 2015 y particularmente a partir de la aprobación de la nueva Ley Universitaria se ha puesto en la UNSAAC las bases para el desarrollo de la investigación en los próximos años: el plan general de fortalecimiento de la investigación, los reglamentos y diferentes directivas están orientados a garantizar la adecuada gestión de la investigación, aun reconociendo que estos instrumentos son perfectibles y estarán en constante modificación.

3.5. Uno de los grandes avances en la gestión de la investigación en la UNSAAC ha sido la creación de plataformas para el acceso a la información e interacción directa en los procesos de convocatoria, difusión y transparencia de todos los actos, eventos, hechos y resultados relacionados a la investigación. Su desarrollo y actualización permanente constituye una tarea útil para posibilitar el desarrollo de la investigación universitaria.

3.6. La utilización pertinente, adecuada y racional de las fuentes de financiamiento, especialmente de los fondos provenientes del canon, constituye un reto para la gestión de la investigación. Existe la necesidad de ampliar el uso de los presupuestos, pero al mismo tiempo garantizar su uso adecuado en términos de pertinencia social de las investigaciones, que deben estar orientados a resolver problemas y necesidades sociales, sin renunciar al desarrollo de los conocimientos y aplicaciones tecnológicas de avanzada.

3.7. Las líneas de investigación que la UNSAAC se ha propuesto a través del Vicerrectorado de Investigación para el periodo 2018-2021 adolecen aún de una excesiva generalidad, se requiere líneas de investigación más cercanas a la problemática social, económica, tecnológica y ambiental que permita plantear proyectos de investigación específicos sin renunciar a la interdisciplinariedad o multidisciplinariedad que la problemática social, económica, tecnológica y ambiental plantea.

3.8. El número de docentes investigadores en la UNSAAC es aún muy bajo, apenas alcanza a un (2.43\% de los profesores nombrados. Se hace necesario incrementar rápida y significativamente el número de docentes investigadores en la Universidad, hasta llegar cuando menos a la proporción de un $20 \%$ de los profesores nombrados. Este hecho significa una profundización de las políticas de investigación, una mayor producción científica y un mejoramiento en la calidad de la formación profesional, además de un crecimiento en la capacidad científica universitaria.

3.9. Las revistas y artículos científicos en la UNSAAC son muy incipientes en número y calidad, apenas 4 revistas de carácter general y un poco menos de 60 artículos indizados. Se hace 
necesario promover la publicación de revistas y artículos científicos indizados, a través de una profundización de las políticas de estímulo a la redacción de artículos y publicación de revistas. Se debe promover la creación de revistas científicas en cada una de las facultades, apoyar su gestión, fortalecimiento e indización a nivel nacional y mundial, para cuyo efecto será fundamental la creación de comités editoriales, capacitación de docentes, creación de plataformas virtuales y calificación por pares externos.

3.10. La creación y publicación del repositorio de tesis de pre y postgrado es un gran avance en la difusión de la producción científica universitaria, sin embargo, es necesario elevar la calidad de las tesis en los aspectos de originalidad, calidad metodológica, calidad de redacción y presentación y sobre todo en la calidad argumentativa, en los cuales la formación científica de los profesores es condición fundamental.

\section{BIBLIOGRAFIA}

UNSAAC (2011) Resolución 010-AU-010-2011-UNSAAC, aprobación de la creación del ViceRectorado de Investigación.

UNSAAC (2015) Estatuto de la Universidad Nacional de San Antonio Abad del Cusco, 2015.

UNSAAC (2016)| Convenio Marco UNSAAC-CONCYTEC-FONDOCYT, del 28 de Noviembre del 2016.

UNSAAC (2017) Compendio Estadístico $\mathrm{N}^{\circ}$ 32, Unidad de Estadística de la Oficina de Planificación Universitaria, Cusco, Perú.

UNSAAC (2017) Líneas de Investigación UNSAAC (2018-2021), Cusco, Perú. Vicerrectorado de Investigación.

UNSAAC (2018) Resolución $\quad N^{\circ}$ CU-561-2018-UNSAAC, Aprueba el Plan General de Fortalecimiento de la Investigación Gestión 2016-2020 "Yachayninchis Wiñarinampaq".

UNSAAC (2019) Boletín Informático "Yachayninchis Wiñaynanpaq"

Gobierno Regional (2005) Ordenanza Regional N 100-2015-GR/GRC-CUSCO, 23, setiembre 2015. Aprobación de la Agenda Regional de Investigación (ARI).

Ordenanza Regional N³0-2012-CR/GRC-Cusco, del 2 de Julio del 2012.

CONCYTEC (2016) Política Nacional para el Desarrollo de la Ciencia, Tecnología e Innovación Tecnológica -CTI.

CONCYTEC (2017) Resolución de Presidencia N| 198-2017-CONCYTEC-P, Aprueba el Reglamento de Calificación y Registro de Investigadores en Ciencia y Tecnología del Sistema Nacional de Ciencia, Tecnología e Innovación Tecnológica -SINACYT.

Ley 2733 (1983) Ley Universitaria Peruana, Perú.

Ley 17437 (1969) Ley Orgánica de la Universidad Peruana

Ley 25203 (1990) Ley del Fondo Especial de Desarrollo Universitario, 23 de febrero de 1990 
Ley N 27506 (2001) Ley Canon, 9 de Julio del 2001.

Ley $N^{\circ} 30220$ (2014) Ley Universitaria, promulgado el 8 de Julio del 2014

RED IDi (2019) Red de Investigación, Desarrollo e Innovación. Recuperado el 24 de Julio del 2019 